\title{
Optimal steering of inertial particles diffusing anisotropically with losses
}

\author{
Yongxin Chen, Tryphon Georgiou and Michele Pavon*
}

October 24, 2018

\begin{abstract}
Exploiting a fluid dynamic formulation for which a probabilistic counterpart might not be available, we extend the theory of Schrödinger bridges to the case of inertial particles with losses and general, possibly singular diffusion coefficient. We find that, as for the case of constant diffusion coefficient matrix, the optimal control law is obtained by solving a system of two p.d.e.'s involving adjoint operators and coupled through their boundary values. In the linear case with quadratic loss function, the system turns into two matrix Riccati equations with coupled split boundary conditions. An alternative formulation of the control problem as a semidefinite programming problem allows computation of suboptimal solutions. This is illustrated in one example of inertial particles subject to a constant rate killing.
\end{abstract}

\section{Introduction}

In 1931/1932, Erwin Schrödinger [1], [2, Section VII] posed the following problem: a large number of i.i.d. Brownian particles in $\mathbb{R}^{n}$ is observed at

${ }^{*}$ Y. Chen and T.T. Georgiou are with the Department of Electrical and Computer Engineering, University of Minnesota, Minneapolis, Minnesota MN 55455, USA, \{chen2468,tryphon\}@umn.edu, and M. Pavon is with the Dipartimento di Matematica, Università di Padova, via Trieste 63, 35121 Padova, Italy, pavon@math.unipd.it 
an initial time $t=0$ to have an empirical distributions $\rho_{0}(x)$, and at a final time $t=T$ an empirical distribution $\rho_{T}(x)$. Assuming that the final distribution differs from the one dictated by the law of large numbers, Schrödinger sought the most likely intermediate empirical distribution for the particle trajectories. He computed that this in fact has one-time density of the form

$$
\rho(x, t)=\varphi(x, t) \hat{\varphi}(x, t)
$$

where $\varphi_{t}$ and $\hat{\varphi}$ are suitable harmonic and co-harmonic functions; i.e., they satisfy

$$
\begin{aligned}
& \frac{\partial \varphi(x, t)}{\partial t}+\frac{1}{2} \sum_{i, j=1}^{n} a_{i j} \frac{\partial^{2}(\hat{\varphi}(x, t))}{\partial x_{i} \partial x_{j}}=0, \\
& \frac{\partial \hat{\varphi}(x, t)}{\partial t}-\frac{1}{2} \sum_{i, j=1}^{n} \frac{\partial^{2}\left(a_{i j} \hat{\varphi}(x, t)\right)}{\partial x_{i} \partial x_{j}}=0 \text {, with } \\
& \varphi(x, 0) \hat{\varphi}(x, 0)=\rho_{0}(x) \text { and } \varphi(x, T) \hat{\varphi}(x, T)=\rho_{T}(x) .
\end{aligned}
$$

As usual, $a=\left(a_{i j}\right)$ represents a constant, positive definite diffusion coefficient (matrix); (1a-1c) is known as a Schrödinger system. Great many insights and generalizations followed as well connections with Quantum mechanics and, specifically, with Schrödinger's own famous equation (see Wakolbinger [3] for a historical account until 1991). The process sought by Schrödinger, as well as the corresponding measure on path space that forms a bridge between beginning and ending marginals, now bear his name. In modern terms, the Schrödinger problem seeks to minimize relative entropy (Kullback-Leibler distance) between distributions on trajectories given the initial and final marginals [4].

Soon afterwards, it became apparent that Schrödinger's bridge has a reformulation as a stochastic optimal control problem, namely to seek a minimum energy control input $u(t)$ so that the diffusion

$$
d X(t)=u(X(t), t) d t+\sigma d w(t)
$$

with $X(0)=\xi$ a.s., $\xi$ distributed according to $\rho_{0}(x)$ and $w(\cdot)$ a standard Wiener process, is consistent with the empirical marginal $\rho_{T}(x)$. Letting 
$a=\sigma \sigma^{\prime}$, the Schrödinger bridge can be constructed by solving

$$
\begin{aligned}
& \inf _{(\rho, u)} \int_{\mathbb{R}^{n}} \int_{0}^{T} \frac{1}{2} u(x, t)^{\prime} a^{-1} u(x, t) \rho(x, t) d t d x, \\
& \frac{\partial \rho}{\partial t}+\nabla \cdot(u \rho)=\frac{1}{2} \sum_{i, j=1}^{n} \frac{\partial^{2}\left(a_{i j} \rho\right)}{\partial x_{i} \partial x_{j}}, \\
& \rho(0, x)=\rho_{0}(x), \quad \rho(T, y)=\rho_{T}(y) .
\end{aligned}
$$

In fact, the optimizing control turns out to be

$$
u^{*}(x, t)=a \nabla \log \varphi(x, t)
$$

where $\varphi(x, t)$ is the space-time harmonic function in the solution of the Schrödinger system. The function $\varphi$ is connected to the neutron importance function which has an important role in perturbation theory and reactor dynamic calculations [5]. The optimal control interpretation relates directly to the property that the Schrödinger bridge represents the law which is closest to the prior measure in the sense of relative entropy; this follows from Girsanov's theory ([4, 6, 7], see also [3] and the references therein).

Interestingly, the connection between Schrödinger bridges and stochastic optimal control seems to have only focused on the case of non-degenerate diffusions, possibly with creation and killing [8, 3, 9], in which the constant diffusion matrix $a$ is nonsingular (to some degree necessitated by the Girsanov theory). Thus, for instance, models of inertial particles driven by stochastic forces do not fall in this category. The authors were initially motivated by this precise problem, to steer inertial particles, and by the possibility of "cooling" oscillators via active feedback for high resolution measuring instruments [10, 11]. In both of these applications, where the stochastic excitation impacts only certain direction of the state vector, i.e., where $a$ is singular, it is still possible to construct generalized Schrödinger bridges for (degenerate) diffusion processes and directly connect to an optimal control problem in a similar fashion. This is done in [12, 13] focusing on linear dynamics and in [14] in a more general context of nonlinear diffusions.

Herein, following [12, 13, we pursue yet another generalization of great practical significance to general diffusion processes. Anisotropic diffusions are important, for instance, in image processing and computer vision [15, 16]. Notice that for general diffusion processes where $\sigma=\sigma(x, t)$ viz. $a=a(x, t)$ 
depends, besides on time, on the spatial variables, a probabilistic problem might not exist. Indeed, for different drift terms, the corresponding distributions on the trajectories may be mutually singular and relative entropy be always infinite. An exception is when the control enters through the "same channel" as the noise [17, (5.3)], [12] or the diffusion coefficient is uniformly bounded, nonsingular and bounded away from zero [18, p.305]. The fluid dynamic formulation (2a, 2c), which resembles the celebrated Benamou-Brenier formulation of the optimal transport problem [19, 20, 21], however, does make sense also in the case of a general diffusion coefficient. More specifically we consider a cloud of particles with density $\rho(x, t), x \in \mathbb{R}^{n}$, which evolves according to the transport-diffusion equation

$$
\frac{\partial \rho}{\partial t}+\nabla \cdot(f(x, t) \rho)+V(x, t) \rho=\frac{1}{2} \sum_{i, j=1}^{n} \frac{\partial^{2}\left(a_{i j}(x, t) \rho\right)}{\partial x_{i} \partial x_{j}}
$$

with $\rho(x, 0)=\rho_{0}$ a probability density. In departure from prior works on connections to Feynman-Kac [3, 7] and in accordance with our aim to be able to model inertial particles, we assume that the matrix $a(x, t)=\left[a_{i j}(x, t)\right]_{i, j=1}^{n}$ is positive semidefinite of constant rank on all of $\mathbb{R}^{n} \times[0, T]$ with

$$
a_{i j}(x, t)=\sum_{k} \sigma_{i k}(x, t) \sigma_{k j}(x, t)
$$

for a matrix $\sigma(x, t)=\left[\sigma_{i k}(x, t)\right] \in \mathbb{R}^{n \times m}$ of constant rank $m \leq n$. The presence of $V(x, t) \geq 0$ allows for the possibility of loss of mass, so that the integral of $\rho(x, t)$ over $\mathbb{R}^{n}$ is not necessarily constant. This flexibility allows modeling the situation where particles, obeying

$$
d X(t)=f(X(t), t) d t+\sigma(X(t), t) d w(t),
$$

are absorbed at some rate by the medium in which they travel or, if the sign of $V$ is negative, created out of this same medium [22, p.272]. We assume here and throughout the paper that $f$ and $\sigma$ are smooth and that the operator

$$
L=\sum_{i, j=1}^{n} a_{i j}(x, t) \partial_{x_{i}} \partial_{x_{j}}+\sum_{j=1}^{n} f_{j}(x, t) \partial_{x_{j}}-\partial_{t}
$$

satisfies Hörmander's condition [23, 24] and is therefore hypoelliptic. Hypoelliptic diffusions occur in many branches of science: Ornstein-Uhlenbeck 
stochastic oscillators, Nyquist-Johnson circuits with noisy resistors, in image reconstruction based on Petitot's model of neurogeometry of vision [25], etc. The "reweighing" the original measure of the Markov process (4) when $V$ is unbounded is a delicate issue and can be accomplished via the Nagasawa transformation, see [3, Section 8B] for the details. We suppose that (3) represents a prior evolution and that at some point $T>0$ we measure an empirical probability density $\rho_{T}(x) \neq \rho(x, T)$ as dictated by (3). Thus, the model (3) is not consistent with the estimated end-point empirical distribution. However, we have reasons to believe that the actual evolution must have been close to the nominal one and that only the actual drift field may be different and equal to

$$
\tilde{f}(x, t):=f(x, t)+\sigma(x, t) u(x, t) .
$$

Notice that the control variables, which may be fewer than $n$, act through the same channels of the diffusive part. The assumption that stochastic excitation and control enter through the same "channels" is natural in certain applications as explained and treated in [12 for linear diffusions. The case were these channels may differ is considered in [13].

The paper is organized as follows: The basic theory is outlined in Section 2 where we derive a generalized Schrödinger system for the optimal control law. We then specialize to the case of linear dynamics with constant diffusion coefficient and quadratic potential $V$ in Section 3 and derive a system of coupled Riccati equations that correspond to the Schrödinger system. The theory, even in the absence of a "killing" potential $V$, falls outside the setting of "linear-quadratic regulator theory" where only one matrix Riccati equation occurs with a specified boundary value. Moreover, here the coupling between the two differential Riccati equations through their split boundary conditions is non-standard, and the solutions to the Riccati equations are sign-indefinite in general [12]. Then, in Section 4 we outline a numerical scheme to obtain suboptimal solutions for the corresponding stochastic control problem. Finally, in Section 5 we conclude with a numerical example.

\section{A generalized Schrödinger system}

Taking (3) as a reference evolution and given the terminal probability density 
$\rho_{T}$, we are led to consider the problem

$$
\begin{aligned}
& \inf _{(\tilde{\rho}, \tilde{u})} \int_{\mathbb{R}^{n}} \int_{0}^{T}\left[\frac{1}{2}\|u\|^{2}+V(x, t)\right] \tilde{\rho}(x, t) d t d x, \\
& \frac{\partial \tilde{\rho}}{\partial t}+\nabla \cdot((f+\sigma u) \tilde{\rho})=\frac{1}{2} \sum_{i, j=1}^{n} \frac{\partial^{2}\left(a_{i j} \tilde{\rho}\right)}{\partial x_{i} \partial x_{j}} \\
& \tilde{\rho}(0, x)=\rho_{0}(x), \quad \tilde{\rho}(T, y)=\rho_{T}(y) .
\end{aligned}
$$

The motivation for this specific form of the index comes from a relative entropy problem on path space (Schrödinger Bridge Problem) in the case when $\left[a_{i j}\right]$ does not depend on the spatial variable $x$ [4, 3, 9]. When $\left[a_{i j}\right]$ does depend on $x$, such an interpretation is available only under rather restrictive assumptions such as uniform boundness of $a$ [17, Section 5]. Problem (5) can therefore be viewed as a generalization of the classical probabilistic Schrödinger bridges problem.

The variational analysis for (5) can be carried out as follows. Let $\mathcal{X}_{\rho_{0} \rho_{T}}$ be the family of flows of probability densities

$$
\tilde{\rho}=\{\tilde{\rho}(\cdot, t) \mid 0 \leq t \leq T\}
$$

satisfying (5c). Let $\mathcal{U}$ be the family of continuous feedback control laws $u(\cdot, \cdot)$. Consider the unconstrained minimization of the Lagrangian over $\mathcal{X}_{\rho_{0} \rho_{T}} \times \mathcal{U}$

$$
\begin{aligned}
\mathcal{L}(\tilde{\rho}, u, \lambda)=\int_{\mathbb{R}^{n}} \int_{0}^{T} & {\left[\left(\frac{1}{2}\|u(x, t)\|^{2}+V(x, t)\right) \tilde{\rho}(x, t)\right.} \\
& -\lambda(x, t)\left(\frac{\partial \tilde{\rho}}{\partial t}+\nabla \cdot((f+\sigma u) \tilde{\rho})\right. \\
- & \left.\left.\frac{1}{2} \sum_{i, j=1}^{n} \frac{\partial^{2}}{\partial x_{i} \partial x_{j}}\left(a_{i j}(x, t) \tilde{\rho}\right)\right)\right] d t d x
\end{aligned}
$$

where $\lambda$ is a $C^{1}$ Lagrange multiplier. After integration by parts, assuming that limits for $x \rightarrow \infty$ are zero, and observing that the boundary values are constant over $\mathcal{X}_{\rho_{0} \rho_{T}}$, we get the problem

$$
\begin{aligned}
& \inf _{(\tilde{\rho}, u) \in \mathcal{X}_{\rho_{0} \rho_{T}} \times \mathcal{U}} \int_{\mathbb{R}^{n}} \int_{0}^{T}\left[\frac{1}{2}\|u\|^{2}+V+\left(\frac{\partial \lambda}{\partial t}\right.\right. \\
& \left.\left.+(f+\sigma u) \cdot \nabla \lambda+\frac{1}{2} \sum_{i, j=1}^{n} a_{i j} \frac{\partial^{2} \lambda}{\partial x_{i} \partial x_{j}}\right)\right] \tilde{\rho}(x, t) d t d x
\end{aligned}
$$


Pointwise minimization of the integrand with respect to $u$ for each fixed flow of probability densities $\tilde{\rho}$ gives

$$
u_{\tilde{\rho}}^{*}(x, t)=-\sigma^{\prime} \nabla \lambda(x, t) .
$$

Plugging this form of the optimal control into (6), we get the functional of $\tilde{\rho} \in \mathcal{X}_{\rho_{0} \rho_{T}}$

$$
\begin{array}{r}
J(\tilde{\rho}, \lambda)=\int_{\mathbb{R}^{n}} \int_{0}^{T}\left[\frac{\partial \lambda}{\partial t}+f \cdot \nabla \lambda-\frac{1}{2} \nabla \lambda \cdot a \nabla \lambda\right. \\
\left.+V+\frac{1}{2} \sum_{i, j=1}^{n} a_{i j}(x, t) \frac{\partial^{2} \lambda}{\partial x_{i} \partial x_{j}}\right] \tilde{\rho}(x, t) d t d x .
\end{array}
$$

We then have the following result:

Proposition 1 If $\tilde{\rho}^{*}$ satisfies

$$
\frac{\partial \tilde{\rho}}{\partial t}+\nabla \cdot((f-a \nabla \lambda) \tilde{\rho})=\frac{1}{2} \sum_{i, j=1}^{n} \frac{\partial^{2}\left(a_{i j} \tilde{\rho}\right)}{\partial x_{i} \partial x_{j}},
$$

with $\lambda$ a solution of the HJB-like equation

$$
\frac{\partial \lambda}{\partial t}+f \cdot \nabla \lambda+\frac{1}{2} \sum_{i, j=1}^{n} a_{i j}(x, t) \frac{\partial^{2} \lambda}{\partial x_{i} \partial x_{j}}=\frac{1}{2} \nabla \lambda \cdot a \nabla \lambda-V
$$

and $\tilde{\rho}^{*}(x, T)=\rho_{T}(x)$, then the pair $\left(\tilde{\rho}^{*}, u^{*}\right)$ with $u^{*}=-\sigma^{\prime} \nabla \lambda$ is a solution of (5).

Of course, the difficulty lies with the nonlinear equation (11) for which no boundary value is available. Together, $\tilde{\rho}(x, t)$ and $\lambda(x, t)$ satisfy the coupled equations 10,11$)$ and the split boundary conditions for $\tilde{\rho}(x, t)$ in $(5 \mathrm{c})$. However, let us define

$$
\varphi(x, t)=\exp [-\lambda(x, t)], \quad(x, t) \in \mathbb{R}^{n} \times[0, T] .
$$

If $\lambda$ satisfies (11), we get that $\varphi$ satisfies the linear equation

$$
\frac{\partial \varphi}{\partial t}+f \cdot \nabla \varphi+\frac{1}{2} \sum_{i, j=1}^{n} a_{i j}(x, t) \frac{\partial^{2} \varphi}{\partial x_{i} \partial x_{j}}=V \varphi
$$


Moreover, for $\tilde{\rho}$ satisfying (10) and $\varphi$ satisfying (12), let us define

$$
\hat{\varphi}(x, t)=\frac{\tilde{\rho}(x, t)}{\varphi(x, t)}, \quad(x, t) \in \mathbb{R}^{n} \times[0, T] .
$$

Then a long but straightforward calculation shows that $\hat{\varphi}$ satisfies the original equation (3). Thus, we have the system of linear PDE's

$$
\begin{aligned}
\frac{\partial \varphi}{\partial t}+f(x, t) \cdot \nabla \varphi+\frac{1}{2} \sum_{i, j=1}^{n} a_{i j} \frac{\partial^{2} \varphi}{\partial x_{i} \partial x_{j}} & =V \varphi, \\
\frac{\partial \hat{\varphi}}{\partial t}+\nabla \cdot(f(x, t) \hat{\varphi})-\frac{1}{2} \sum_{i, j=1}^{n} \frac{\partial^{2}\left(a_{i j} \hat{\varphi}\right)}{\partial x_{i} \partial x_{j}} & =-V \hat{\varphi},
\end{aligned}
$$

nonlinearly coupled through their boundary values as

$$
\varphi(x, 0) \hat{\varphi}(x, 0)=\tilde{\rho}_{0}(x), \quad \varphi(x, T) \hat{\varphi}(x, T)=\tilde{\rho}_{T}(x) .
$$

Equations 13a)-13c constitute a generalized Schrödinger system. We have therefore established the following result.

Theorem 1 Let $(\varphi(x, t), \hat{\varphi}(x, t))$ be nonnegative functions satisfying (13a)(13c) for $(x, t) \in\left(\mathbb{R}^{n} \times[0, T]\right)$. Suppose $\varphi$ is everywhere positive. Then the pair $\left(\tilde{\rho}^{*}, u^{*}\right)$ with

$$
\begin{aligned}
u^{*}(x, t) & =\sigma^{\prime} \nabla \log \varphi(x, t), \\
\frac{\partial \tilde{\rho}}{\partial t}+\nabla \cdot((f+a \nabla \log \varphi) \tilde{\rho}) & =\frac{1}{2} \sum_{i, j=1}^{n} \frac{\partial^{2}\left(a_{i j} \tilde{\rho}\right)}{\partial x_{i} \partial x_{j}}
\end{aligned}
$$

is a solution of (5).

Establishing existence and uniqueness (up to multiplication/division of the two functions by a positive constant) of the solution of the Schrödinger system is extremely challenging even when the diffusion coefficient matrix $a$ is constant and nonsingular. Nevertheless, if the fundamental solution $p$ of $(3)$ is everywhere positive on $\left(\mathbb{R}^{n} \times(0, T]\right)$, existence and uniqueness follows from a deep result of Beurling [26] suitably extended by Jamison [27, Theorem 3.2], [3, Section 10]. 
Remark 1 It is interesting to note that although (5) is not convex in $(\tilde{\rho}, u)$, it can be turned into a convex problem in a new set of coordinates $(\tilde{\rho}, \tilde{m})$ where $m=\tilde{\rho} u$, in which case it becomes

$$
\begin{aligned}
& \inf _{(\tilde{\rho}, \tilde{m})} \int_{\mathbb{R}^{n}} \int_{0}^{T}\left[\frac{1}{2} \frac{\|m\|^{2}}{\tilde{\rho}(x, t)}+V(x, t) \tilde{\rho}(x, t)\right] d t d x, \\
& \frac{\partial \tilde{\rho}}{\partial t}+\nabla \cdot(f \tilde{\rho}+\sigma m)=\frac{1}{2} \sum_{i, j=1}^{n} \frac{\partial^{2}\left(a_{i j} \tilde{\rho}\right)}{\partial x_{i} \partial x_{j}} \\
& \tilde{\rho}(0, x)=\rho_{0}(x), \quad \tilde{\rho}(T, y)=\rho_{T}(y) .
\end{aligned}
$$

This type of coordinate transformation has been effectively used in [19] in the context of optimal mass transport.

\section{The linear-quadratic case}

We now specialize system 13 to the case of linear dynamics with constant diffusion matrix and quadratic loss function $V(x)$, i.e., we assume that $\rho(x, t)$ represents the density function of a linear diffusion

$$
d X(t)=A X(t) d t+B u(t)+B d w(t), \text { with } X(0)=\xi, \text { a.s. }
$$

and $\xi$ distributed according to

$$
\rho(x, 0)=\frac{1}{\sqrt{(2 \pi)^{n} \operatorname{det}\left(\Sigma_{0}\right)}} \exp \left(-\frac{1}{2} x^{\prime} \Sigma_{0}^{-1} x\right)
$$

with $\Sigma_{0}>0$. We also assume a loss/state-cost function

$$
V(x, t)=\frac{1}{2} x^{\prime} S(t) x
$$

and a "target" end-point distribution

$$
\rho(x, T)=\frac{1}{\sqrt{(2 \pi)^{n} \operatorname{det}\left(\Sigma_{T}\right)}} \exp \left(-\frac{1}{2} x^{\prime} \Sigma_{T}^{-1} x\right),
$$

at $t=T$, with $S(t) \geq 0$ and $\Sigma_{T}>0 \square^{1}$.

\footnotetext{
${ }^{1}$ The special case where the diffusion coefficient $B B^{\prime}$ is positive definite and $V \equiv 0$ has been studied in $[28$.
} 
Take $\varphi(x, t)$ and $\hat{\varphi}(x, t)$ in the form

$$
\begin{aligned}
& \varphi(x, t)=c(t) \exp \left\{-\frac{1}{2} x^{\prime} \Pi(t) x\right\} \\
& \hat{\varphi}(x, t)=\hat{c}(t) \exp \left\{-\frac{1}{2} x^{\prime} \mathrm{H}(t) x\right\} .
\end{aligned}
$$

Substitution into (13) and separation of variables leads after straightforward calculation to the following two coupled Riccati equations with split boundary conditions

$$
\begin{aligned}
-\dot{\Pi}(t) & =A^{\prime} \Pi(t)+\Pi(t) A-\Pi(t) B B^{\prime} \Pi(t)+S(t) \\
-\dot{\mathrm{H}}(t) & =A^{\prime} \mathrm{H}(t)+\mathrm{H}(t) A+\mathrm{H}(t) B B^{\prime} \mathrm{H}(t)-S(t)
\end{aligned}
$$

with

$$
\Sigma_{0}^{-1}=\Pi(0)+\mathrm{H}(0) \text { and } \Sigma_{T}^{-1}=\Pi(T)+\mathrm{H}(T) .
$$

and

$$
\begin{aligned}
& c(t)=\exp \left\{\frac{1}{2} \int_{0}^{t} \operatorname{trace}\left(B B^{\prime} \Pi(\tau)\right) d \tau\right\} \\
& \hat{c}(t)=\exp \left\{-\int_{0}^{t} \operatorname{trace}\left[A(\tau)+\frac{1}{2} B B^{\prime} \mathrm{H}(\tau)\right] d \tau\right\}
\end{aligned}
$$

Thus, the problem reduces to finding a pair $(\Pi(t), \mathrm{H}(t))$ satisfying (17). For the case when $S(t) \equiv 0$, it has been shown by the authors in [12] that this system has a unique solution.

\section{Semi-definite programming formulation}

It appears that the solution of $(17 \mathrm{a}-17 \mathrm{c})$ using successive approximation is not numerically stable. Thus, we now present an alternative formulation into semi-definite program. This allows computation of suboptimal solutions that are arbitrarily close to being optimal. This method was used to work out the example that follows in Section 5 . 
We are interested in computing a feedback gain $K(t)$ so that the control signal $u(t)=-K(t) x(t)$ steers (16) from the initial state-covariance $\Sigma_{0}$ at $t=0$ to the final $\Sigma_{T}$ at $t=T$. The cost functional to be minimized is

$$
\begin{aligned}
J & =\mathbb{E}\left\{\int_{0}^{T}\left[\frac{1}{2}\|u\|^{2}+\frac{1}{2} x(t)^{\prime} S(t) x(t)\right] d t\right\} \\
& =\frac{1}{2} \int_{0}^{T}\left[\operatorname{trace}\left(K(t) \Sigma(t) K(t)^{\prime}\right)+\operatorname{trace}(S(t) \Sigma(t))\right] d t
\end{aligned}
$$

subject to the corresponding differential Lyapunov equation for the state covariance

$$
\dot{\Sigma}(t)=(A-B K) \Sigma(t)+\Sigma(t)(A-B K)^{\prime}+B B^{\prime}
$$

satisfying the boundary conditions

$$
\Sigma(0)=\Sigma_{0}, \text { and } \Sigma(T)=\Sigma_{T} .
$$

If we replace $K$ by $U(t):=-\Sigma(t) K(t)^{\prime}$, then

$$
J=\frac{1}{2} \int_{0}^{T}\left[\operatorname{trace}\left(U(t)^{\prime} \Sigma(t)^{-1} U(t)\right)+\operatorname{trace}(S(t) \Sigma(t))\right] d t
$$

becomes jointly convex in $U(t)$ and $\Sigma(t)$. On the other hand, the Lyapunov equation (19) becomes

$$
\dot{\Sigma}(t)=A \Sigma(t)+\Sigma(t) A^{\prime}+B U(t)^{\prime}+U(t) B^{\prime}+B B^{\prime}
$$

and is now linear in both $U$ and $\Sigma$. Thus, our optimization problem reduces to the semi-definite program to minimize

$$
\int_{0}^{T}[\operatorname{trace}(Y(t))+\operatorname{trace}(S(t) \Sigma(t))] d t
$$

subject to 20a 20b and

$$
\left[\begin{array}{cc}
Y(t) & U(t)^{\prime} \\
U(t) & \Sigma(t)
\end{array}\right] \geq 0
$$

After discretization in time, 20a 20d can be solved numerically and a (suboptimal) gain recovered as

$$
K(t)=-U(t)^{\prime} \Sigma(t)^{-1} .
$$




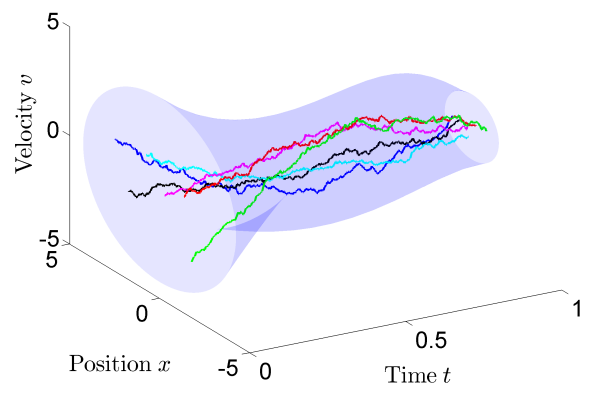

Figure 1: Inertial particles: state trajectories $(S(t) \equiv I)$

\section{Example}

We consider inertial particles modeled by

$$
\begin{aligned}
& d x(t)=v(t) d t \\
& d v(t)=u(t) d t+d w(t) .
\end{aligned}
$$

Here, $u(t)$ is a control input (force) at our disposal, $x(t)$ represents the position and $v(t)$ velocity of particles, while $w(t)$ represents random exitation (corresponding to "white noise" forcing). We wish to steer the spread of the particles from an initial Gaussian distribution with $\Sigma_{0}=2 I$ at $t=0$ to the terminal marginal $\Sigma_{T}=1 / 4 I$ for $T=1$ in a optimal way such that the cost function 18 is minimized.

Figure 1 displays typical sample paths $\{(x(t), v(t)) \mid t \in[0,1]\}$ in phase space, as a function of time, that are attained using the optimal feedback strategy derived following (20c) and $S=I$. The feedback gains $K(t)=$ $\left[k_{1}(t), k_{2}(t)\right]$ are shown in Figure 2 as a function of time. Figure 3 shows the corresponding control action for each trajectory.

For comparison, Figure 4 displays typical sample paths when optimal control is used and $S=10 I$. As expected, $\Sigma(\cdot)$ shrinks faster as we increase the state penalty $S$ which is consistent with the reference evolution loosing probability mass at a higher rate at places where $V(x)$ is large. 


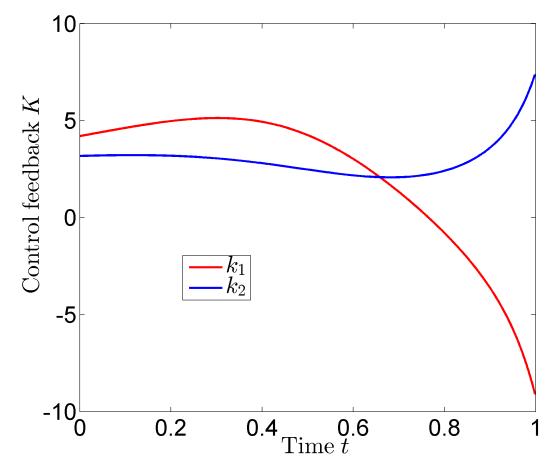

Figure 2: Inertial particles: feedback gains

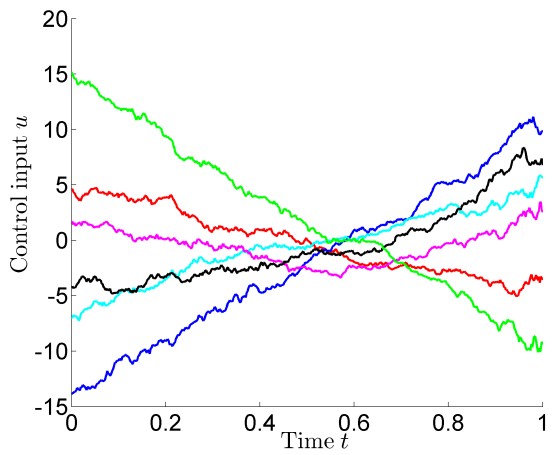

Figure 3: Inertial particles: control inputs

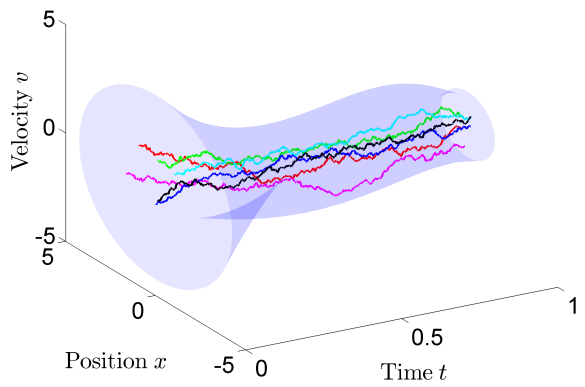

Figure 4: Inertial particles: state trajectories $(S(t) \equiv 10 I)$ 


\section{References}

[1] E. Schrödinger, Über die umkehrung der naturgesetze. Verlag Akademie der wissenschaften in kommission bei Walter de Gruyter u. Company, 1931.

[2] — - "Sur la théorie relativiste de l'électron et l'interprétation de la mécanique quantique," in Annales de l'institut Henri Poincaré, vol. 2, no. 4. Presses universitaires de France, 1932, pp. 269-310.

[3] A. Wakolbinger, "Schrödinger bridges from 1931 to 1991," in Proc. of the 4th Latin American Congress in Probability and Mathematical Statistics, Mexico City, 1990, pp. 61-79.

[4] H. Föllmer, "Random fields and diffusion processes," in École d'Été de Probabilités de Saint-Flour XV-XVII, 1985-87. Springer, 1988, pp. 101-203.

[5] S. Feghhi, M. Shahriari, and H. Afarideh, "Calculation of neutron importance function in fissionable assemblies using Monte Carlo method," Annals of Nuclear Energy, vol. 34, no. 6, pp. 514-520, 2007.

[6] P. Dai Pra, "A stochastic control approach to reciprocal diffusion processes," Applied mathematics and Optimization, vol. 23, no. 1, pp. 313329, 1991.

[7] M. Pavon and A. Wakolbinger, "On free energy, stochastic control, and Schrödinger processes," in Modeling, Estimation and Control of Systems with Uncertainty. Springer, 1991, pp. 334-348.

[8] D. Dawson, L. Gorostiza, and A. Wakolbinger, "Schrödinger processes and large deviations," Journal of mathematical physics, vol. 31, no. 10, pp. 2385-2388, 1990.

[9] P. Dai Pra and M. Pavon, "On the Markov processes of Schrödinger, the Feynman-Kac formula and stochastic control," in Realization and Modelling in System Theory. Springer, 1990, pp. 497-504.

[10] A. Vinante, M. Bignotto, M. Bonaldi, M. Cerdonio, L. Conti, P. Falferi, N. Liguori, S. Longo, R. Mezzena, A. Ortolan et al., "Feedback cooling of the normal modes of a massive electromechanical system to submil- 
likelvin temperature," Physical review letters, vol. 101, no. 3, p. 033601, 2008.

[11] T. Munakata and M. Rosinberg, "Feedback cooling, measurement errors, and entropy production," Journal of Statistical Mechanics: Theory and Experiment, vol. 2013, no. 06, p. P06014, 2013.

[12] Y. Chen, T. Georgiou, and M. Pavon, "Optimal steering of a linear stochastic system to a final probability distribution," arXiv preprint arXiv:1408.2222, 2014.

[13] — - "Optimal steering of a linear stochastic system to a final probability distribution, part II," in preparation.

[14] _ _ "Fast cooling for a system of stochastic oscillators," in preparation.

[15] P. Perona and J. Malik, "Scale-space and edge detection using anisotropic diffusion," Pattern Analysis and Machine Intelligence, IEEE Transactions on, vol. 12, no. 7, pp. 629-639, 1990.

[16] P. Guidotti, "Anisotropic diffusions of image processing from PeronaMalik on," Advanced Studies in Pure Mathematics, vol. 99, p. 20XX, 2007.

[17] M. Fischer, "On the form of the large deviation rate function for the empirical measures of weakly interacting systems," Bernoulli, vol. 20, no. 4, pp. 1765-1801, 2014.

[18] I. Karatzas, Brownian motion and stochastic calculus. springer, 1991, vol. 113.

[19] J.-D. Benamou and Y. Brenier, "A computational fluid mechanics solution to the Monge-Kantorovich mass transfer problem," Numerische Mathematik, vol. 84, no. 3, pp. 375-393, 2000.

[20] C. Villani, Topics in optimal transportation. American Mathematical Soc., 2003, no. 58.

[21] L. Ambrosio, N. Gigli, and G. Savaré, Gradient flows: in metric spaces and in the space of probability measures. Springer, 2006.

[22] S. Karlin and H. M. Taylor, A second course in stochastic processes. 
Gulf Professional Publishing, 1981, vol. 2.

[23] L. Hörmander, "Hypoelliptic second order differential equations," Acta Mathematica, vol. 119, no. 1, pp. 147-171, 1967.

[24] O. A. Oleinik and E. V. Radkevich, "Second order equations with nonnegative characteristic form," Itogi Nauki i Tekhniki. Seriya" Matematicheskii Analiz", vol. 7, pp. 7-252, 1971.

[25] U. Boscain, R. Chertovskih, J.-P. Gauthier, and A. Remizov, "Hypoelliptic diffusion and human vision: A semidiscrete new twist," SIAM Journal on Imaging Sciences, vol. 7, no. 2, pp. 669-695, 2014.

[26] A. Beurling, "An automorphism of product measures," The Annals of Mathematics, vol. 72, no. 1, pp. 189-200, 1960.

[27] B. Jamison, "Reciprocal processes," Z. Wahrscheinlichkeitstheorie verw. Gebiete, vol. 30, pp. 65-86, 1974.

[28] I. G. Vladimirov and I. R. Petersen, "Minimum relative entropy state transitions in linear stochastic systems: the continuous time case," in Proceedings of 19th International Symposium on Mathematical Theory of Networks and Systems, 2010, pp. 51-58. 